\title{
Baryon parity doublets and chiral spin symmetry
}

\author{
M. Catillo and L. Ya. Glozman \\ Institute of Physics, University of Graz, 8010 Graz, Austria
}

(Received 24 April 2018; published 25 July 2018)

\begin{abstract}
The chirally symmetric baryon parity-doublet model can be used as an effective description of the baryon-like objects in the chirally symmetric phase of QCD. Recently it has been found that above the critical temperature, higher chiral spin symmetries emerge in QCD. It is demonstrated here that the baryon parity-doublet Lagrangian is manifestly chiral spin invariant. We construct nucleon interpolators with fixed chiral spin transformation properties that can be used in lattice studies at high $\mathrm{T}$.
\end{abstract}

DOI: 10.1103/PhysRevD.98.014030

\section{BARYON PARITY DOUBLETS. INTRODUCTION}

A Dirac Lagrangian of a massless fermion field is chirally symmetric since the left- and right-handed components of the fermion field are decoupled,

$$
\mathcal{L}=i \bar{\psi} \gamma_{\mu} \partial^{\mu} \psi=i \bar{\psi}_{L} \gamma_{\mu} \partial^{\mu} \psi_{L}+i \bar{\psi}_{R} \gamma_{\mu} \partial^{\mu} \psi_{R}
$$

where

$$
\psi_{R}=\frac{1}{2}\left(1+\gamma_{5}\right) \psi, \quad \psi_{L}=\frac{1}{2}\left(1-\gamma_{5}\right) \psi
$$

The $S U(2)_{L} \times S U(2)_{R}$ chiral symmetry is a symmetry upon independent isospin rotations of the left- and righthanded components of fermions,

$\psi_{R} \rightarrow \exp \left(l \frac{\theta_{R}^{a} \tau^{a}}{2}\right) \psi_{R} ; \quad \psi_{L} \rightarrow \exp \left(l \frac{\theta_{L}^{a} \tau^{a}}{2}\right) \psi_{L}$,

where $\tau^{a}$ are the isospin Pauli matrices and the angles $\theta_{R}^{a}$ and $\theta_{L}^{a}$ parametrize rotations of the right- and left-handed components. The transformation (3) defines the $(0,1 / 2) \oplus$ $(1 / 2,0)$ representation of the chiral group.

The mass term in the Lagrangian breaks explicitly the chiral symmetry since it couples the left- and right-handed fermions.

It was known for a long time that it is possible to construct a chirally symmetric Lagrangian for a massive fermion field provided that there are mass-degenerate

Published by the American Physical Society under the terms of the Creative Commons Attribution 4.0 International license. Further distribution of this work must maintain attribution to the author(s) and the published article's title, journal citation, and DOI. Funded by SCOAP. fermions of opposite parity, parity doublets, that transform into each other upon a chiral transformation [1].

Consider a pair of the isodoublet fermion fields

$$
\Psi=\left(\begin{array}{l}
\Psi_{+} \\
\Psi_{-}
\end{array}\right)
$$

where the Dirac bispinors $\Psi_{+}$and $\Psi_{-}$have positive and negative parity, respectively. The parity doublet above is a spinor constructed from two Dirac bispinors and contains eight components. Note that there is, in addition, an isospin index which is suppressed. Given that the right- and lefthanded fields are directly connected with the opposite parity fields

$$
\Psi_{R}=\frac{1}{\sqrt{2}}\left(\Psi_{+}+\Psi_{-}\right) ; \quad \Psi_{L}=\frac{1}{\sqrt{2}}\left(\Psi_{+}-\Psi_{-}\right),
$$

the vectorial and axial parts of the chiral transformation law under the $(0,1 / 2) \oplus(1 / 2,0)$ representation of $S U(2)_{L} \times S U(2)_{R}$ is

$$
\begin{aligned}
& \Psi \rightarrow \exp \left(l \frac{\theta_{V}^{a} \tau^{a}}{2} \otimes \mathbb{1}\right) \Psi \\
& \Psi \rightarrow \exp \left(l \frac{\theta_{A}^{a} \tau^{a}}{2} \otimes \sigma_{1}\right) \Psi .
\end{aligned}
$$

Here $\sigma_{i}$ is a Pauli matrix that acts in the space of the parity doublet. In the chiral transformation law (3) the axial rotation mixes the massless Dirac spinor $\psi$ with $\gamma_{5} \psi$, while in the chiral rotation of the parity doublet, a mixing of two independent fields $\Psi_{+}$and $\Psi_{-}$is provided. Then the chiralinvariant Lagrangian of the free parity doublet is given as

$$
\begin{aligned}
\mathcal{L} & =i \bar{\Psi} \gamma^{\mu} \partial_{\mu} \Psi-m \bar{\Psi} \Psi \\
& =i \bar{\Psi}_{+} \gamma^{\mu} \partial_{\mu} \Psi_{+}+i \bar{\Psi}_{-} \gamma^{\mu} \partial_{\mu} \Psi_{-}-m \bar{\Psi}_{+} \Psi_{+}-m \bar{\Psi}_{-} \Psi_{-} .
\end{aligned}
$$


Note that this Lagrangian can be written in different equivalent forms $[2,3]$. The equivalence of the present form [1] with that one of Ref. [3] was demonstrated in Ref. [4] and the chiral transformation law (6) corresponds to the "mirror" assignment of Ref. [3]. For the reader's convenience we derive this equivalence in the Appendix. This Lagrangian can also be equivalently written in terms of the right- and left-handed fields of Eq. (5),

$\mathcal{L}=i \bar{\Psi}_{L} \gamma^{\mu} \partial_{\mu} \Psi_{L}+i \bar{\Psi}_{R} \gamma^{\mu} \partial_{\mu} \Psi_{R}-m \bar{\Psi}_{L} \Psi_{L}-m \bar{\Psi}_{R} \Psi_{R}$.

The latter form demonstrates that the right- and left-handed degrees of freedom are completely decoupled and the Lagrangian is manifestly chiral invariant. Since the latter Lagrangian is equivalent to the Lagrangian (7) it is also manifestly Lorentz invariant [in order to avoid confusion note that $\Psi_{L}$ and $\Psi_{R}$ for the parity doublet are defined differently from the massless Dirac bispinor (2)].

A crucial property of the Lagrangian (7)-(8) is that the fermions $\Psi_{+}$and $\Psi_{-}$are exactly degenerate and have a nonzero chiral-invariant mass $m$. This Lagrangian has a number of peculiarities. In particular, the diagonal axial charge of the fermions $\Psi_{-}$and $\Psi_{+}$vanishes, while the offdiagonal axial charge is 1 .

Supplemented by the interactions with the pion and sigma fields [2,3] this Lagrangian has been sometimes used in baryon spectroscopy [5] and as an effective model for the chiral symmetry restoration scenario at high temperature or density where baryons with the nonzero mass do not vanish upon a chiral restoration [6].

Recently it was found on the lattice [7] that at temperatures above the critical one new symmetries emerge in QCD, $S U(2)_{C S}$, and $S U\left(2 N_{F}\right)$ [8,9], which implies a modification of the existing view on the nature of the strongly interacting matter at high temperatures [10]. In particular, the elementary objects at high temperatures are quarks with a definite chirality connected by the chromoelectric field; i.e., there are no free deconfined quarks.

These symmetries were observed earlier upon artificial truncation of the near-zero modes of the Dirac operator at zero temperature [11-14]; for a recent theoretical development see [15]. The $S U(2)_{C S} \supset U(1)_{A}$ and $S U\left(2 N_{F}\right) \supset$ $S U\left(N_{F}\right)_{L} \times S U\left(N_{F}\right)_{R} \times U(1)_{A} \quad$ symmetries are symmetries of the chromo-electric interaction in QCD. In addition to the chiral transformations, the $S U(2)_{C S}$ and $S U\left(2 N_{F}\right)$ rotations mix the left- and right-handed components of quark fields. The chromo-magnetic interaction as well as the quark kinetic term break these symmetries down to $S U\left(N_{F}\right)_{L} \times S U\left(N_{F}\right)_{R} \times U(1)_{A}$.

Given the observed new symmetries in high T QCD, a question arises of whether the parity-doublet model could still be used as an effective description for the baryon-like objects at high T. Here we demonstrate that this model is actually manifestly $S U(2)_{C S}$ and $S U\left(2 N_{F}\right)$ invariant.

\section{CHIRAL SPIN SYMMETRY}

The $S U(2)_{C S}$ chiral spin transformations and generators $[8,9]$, defined in the Dirac quark spinor space are

$$
\begin{aligned}
\psi \rightarrow \psi^{\prime} & =\exp \left(i \frac{\varepsilon^{n} \Sigma^{n}}{2}\right) \psi, \\
\Sigma^{n} & =\left\{\gamma_{k},-i \gamma_{5} \gamma_{k}, \gamma_{5}\right\},
\end{aligned}
$$

$n=1,2,3$. Here $\gamma_{k}, k=1,2,3,4$, is any Hermitian Euclidean gamma matrix, obeying the following anticommutation relations:

$$
\gamma_{i} \gamma_{j}+\gamma_{j} \gamma_{i}=2 \delta^{i j} ; \quad \gamma_{5}=\gamma_{1} \gamma_{2} \gamma_{3} \gamma_{4} .
$$

Different $k$ defines different four-dimensional representations that can be reduced into irreducible ones of $\operatorname{dim}=2$. The $\mathfrak{s} \mathfrak{u t}(2)$ algebra $\left[\Sigma^{a}, \Sigma^{b}\right]=2 i \epsilon^{a b c} \Sigma^{c}$ is satisfied with any Euclidean gamma matrix.

$U(1)_{A}$ is a subgroup of $S U(2)_{C S}$. The $S U(2)_{C S}$ transformations mix the left- and right-handed fermions. The free massless quark Lagrangian (1) does not have this symmetry.

An extension of the direct $S U(2)_{C S} \times S U\left(N_{F}\right)$ product leads to a $S U\left(2 N_{F}\right)$ group. This group contains the chiral symmetry of QCD $S U\left(N_{F}\right)_{L} \times S U\left(N_{F}\right)_{R} \times U(1)_{A}$ as a subgroup. Its transformations are given by

$$
\psi \rightarrow \psi^{\prime}=\exp \left(i \frac{\epsilon^{m} T^{m}}{2}\right) \psi
$$

where $m=1,2, \ldots,\left(2 N_{F}\right)^{2}-1$ and the set of $\left(2 N_{F}\right)^{2}-1$ generators is

$$
\left\{\left(\tau^{a} \otimes \mathbb{1}_{D}\right),\left(\mathbb{1}_{F} \otimes \Sigma^{n}\right),\left(\tau^{a} \otimes \Sigma^{n}\right)\right\}
$$

with $\tau$ being the flavor generators with the flavor index $a$ and $n=1,2,3$ is the $S U(2)_{C S}$ index.

The fundamental vector of $S U\left(2 N_{F}\right)$ at $N_{F}=2$ is

$$
\psi=\left(\begin{array}{c}
u_{\mathrm{R}} \\
u_{\mathrm{L}} \\
d_{\mathrm{R}} \\
d_{\mathrm{L}}
\end{array}\right) .
$$

The $S U\left(2 N_{F}\right)$ transformations mix both flavor and chirality.

While the $S U(2)_{C S}$ and $S U\left(2 N_{F}\right)$ symmetries are not symmetries of the QCD Lagrangian as a whole, they are symmetries of the fermion charge operator and of the chromo-electric interaction in QCD. The chromo-magnetic interaction as well as the quark kinetic term break these symmetries. 


\section{CHIRAL SPIN SYMMETRY OF THE PARITY DOUBLETS}

The chiral spin transformation (9) and generators (10) at $k=4$ (the Euclidean $\gamma_{4}$ matrix coincides with the Minkowskian $\gamma_{0}$ matrix) can be presented in an equivalent form, as follows below. We will use the chiral representation of the $\gamma$ matrices. Then the $S U(2)_{C S}$ generators for the representation with $k=4$ are

$$
\Sigma^{n}=\left\{\mathbb{1} \otimes \sigma^{1}, \mathbb{1} \otimes \sigma^{2}, \mathbb{1} \otimes \sigma^{3}\right\} .
$$

Here $\mathbb{1}$ is the unit $2 \times 2$ matrix and the Pauli matrices $\sigma^{i}$ act in the space of spinors

$$
\left(\begin{array}{l}
R \\
L
\end{array}\right)
$$

where $R$ and $L$ represent the upper and lower components of the right- and left-handed Dirac bispinors (2)

$$
\begin{aligned}
& \psi_{R}=\left(\begin{array}{l}
R \\
0
\end{array}\right), \\
& \psi_{L}=\left(\begin{array}{l}
0 \\
L
\end{array}\right) .
\end{aligned}
$$

The $S U(2)_{C S}, k=4$ transformation (9) can then be rewritten as

$\psi \rightarrow \psi^{\prime}=\exp \left(i \frac{\varepsilon^{n} \Sigma^{n}}{2}\right) \psi=\exp \left(i \frac{\varepsilon^{n} \sigma^{n}}{2}\right)\left(\begin{array}{l}R \\ L\end{array}\right)$.

The latter equation emphasizes that a reduction of the representations (9) of $S U(2)_{C S}$ leads to a two-dimensional irreducible representation.

Now we return to the parity-doublet Lagrangian. Given Eq. (5), the parity doublet (4) can be unitary transformed into a doublet

$$
\tilde{\Psi}=\left(\begin{array}{c}
\Psi_{R} \\
\Psi_{L}
\end{array}\right)
$$

which is a two-component spinor composed of Dirac bispinors $\Psi_{R}$ and $\Psi_{L}$ (i.e., all together there are eight components).

The Lagrangian (7) is obviously invariant upon the $S U(2)_{C S}$ rotation of the doublet (20),

$$
\left(\begin{array}{c}
\Psi_{R} \\
\Psi_{L}
\end{array}\right) \rightarrow \exp \left(i \frac{\varepsilon^{n} \sigma^{n}}{2}\right)\left(\begin{array}{l}
\Psi_{R} \\
\Psi_{L}
\end{array}\right)
$$

It then follows that the parity-doublet Lagrangian is not only chirally invariant under the transformation (6), but is, in addition, $S U(2)_{C S}$ and $S U\left(2 N_{F}\right)$ invariant with the generators of $S U\left(2 N_{F}\right)$ being

$$
\left\{\left(\tau^{a} \otimes \mathbb{1}\right),\left(\mathbb{1} \otimes \sigma^{n}\right),\left(\tau^{a} \otimes \sigma^{n}\right)\right\} .
$$

\section{CHIRAL SPIN SYMMETRIC NUCLEON INTERPOLATORS}

Here we construct nucleon three-quark interpolators that transform upon $S U(2)_{C S}$ representations (8)-(9) with $k=4$. These interpolators should be used in lattice studies to establish the chiral spin symmetry and consequently to justify the parity-doublet model as an effective description of the baryon-like objects at high temperatures.

The quark bispinor, divided into its $R$ and $L$ components, is

$$
q=\left(\begin{array}{l}
q^{R} \\
q^{L}
\end{array}\right),
$$

where $q=u$ or $d$. It transforms under the representation 2 of $S U(2)_{C S}$. Therefore, the nucleon three-quark interpolators fall into the representation $2 \otimes \mathbf{2} \otimes \mathbf{2}=\mathbf{2}_{\mathbf{1}} \oplus \mathbf{2} \boldsymbol{2} \oplus \mathbf{4}$.

Using the standard technique of Young tableaux for representations of the permutation group

$$
\begin{aligned}
& u_{a_{s}}^{\alpha} d_{b_{j}}^{\beta} u_{c_{k}}^{\gamma}=\alpha \otimes \beta \otimes \gamma=
\end{aligned}
$$

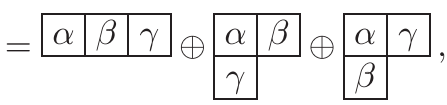

where indices $\alpha, \beta, \gamma$ denote either $L$ or $R$, and, e.g., $u_{a_{s}}^{L}$ is the left part of the up quark with the color index $a$ and the spin index $s$, we get

$$
\begin{aligned}
& \mathbf{2}_{\mathbf{1}}: \begin{array}{|l|l|}
\hline \alpha & \gamma
\end{array} \quad \Rightarrow \epsilon_{\alpha \beta} u_{a_{s}}^{\alpha} d_{b_{j}}^{\beta} u_{c_{k}}^{\gamma} \\
& \mathbf{2}_{2}: \begin{array}{l|l|l}
\alpha & \beta
\end{array} \quad \Rightarrow u_{a_{s}}^{\alpha} d_{b_{j}}^{\beta} u_{c_{k}}^{\gamma}+u_{a_{s}}^{\beta} d_{b_{j}}^{\alpha} u_{c_{k}}^{\gamma}+ \\
& \text { - } u_{a_{s}}^{\alpha} d_{b_{j}}^{\gamma} u_{c_{k}}^{\beta}-u_{a_{s}}^{\gamma} d_{b_{j}}^{\alpha} u_{c_{k}}^{\beta}
\end{aligned}
$$

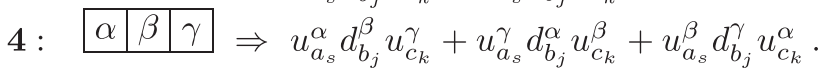

Hence, the nucleon fields in these irreducible representations of $S U(2)_{C S}$ are given by

$$
\mathbf{2}_{1}:\left\{\begin{array}{l}
B_{2_{1}}(-1 / 2)=\frac{1}{\sqrt{2}}\left(u_{a_{s}}^{L} d_{b_{j}}^{R}-u_{a_{s}}^{R} d_{b_{j}}^{L}\right) u_{c_{k}}^{L} \epsilon_{a b c} f(s, j, k), \\
B_{2_{1}}(1 / 2)=\frac{1}{\sqrt{2}}\left(u_{a_{s}}^{L} d_{b_{j}}^{R}-u_{a_{s}}^{R} d_{b_{j}}^{L}\right) u_{c_{k}}^{R} \epsilon_{a b c} f(s, j, k),
\end{array}\right.
$$




$$
\begin{gathered}
\mathbf{2}_{2}:\left\{\begin{array}{l}
B_{2_{2}}(-1 / 2)=\left(\sqrt{\frac{2}{3}} u_{a_{s}}^{L} d_{b_{j}}^{L} u_{c_{k}}^{R}-\sqrt{\frac{1}{6}} u_{a_{s}}^{L} d_{b_{j}}^{R} u_{c_{k}}^{L}-\sqrt{\frac{1}{6}} u_{a_{s}}^{R} d_{b_{j}}^{L} u_{c_{k}}^{L}\right) \epsilon_{a b c} f(s, j, k), \\
B_{2_{2}}(1 / 2)=\left(\sqrt{\frac{1}{6}} u_{a_{s}}^{L} d_{b_{j}}^{R} u_{c_{k}}^{R}+\sqrt{\frac{1}{6}} u_{a_{s}}^{R} d_{b_{j}}^{L} u_{c_{k}}^{R}-\sqrt{\frac{2}{3}} u_{a_{s}}^{R} d_{b_{j}}^{R} u_{c_{k}}^{L}\right) \epsilon_{a b c} f(s, j, k),
\end{array}\right. \\
\mathbf{4 :}\left\{\begin{array}{l}
B_{4}(-3 / 2)=u_{a_{s}}^{L} d_{b_{j}}^{L} u_{c_{k}}^{L} \epsilon_{a b c} f(s, j, k), \\
B_{4}(-1 / 2)=\sqrt{\frac{1}{3}}\left(u_{a_{s}}^{L} d_{b_{j}}^{L} u_{c_{k}}^{R}+u_{a_{s}}^{L} d_{b_{j}}^{R} u_{c_{k}}^{L}+u_{a_{s}}^{R} d_{b_{j}}^{L} u_{c_{k}}^{L}\right) \epsilon_{a b c} f(s, j, k), \\
B_{4}(1 / 2)=\sqrt{\frac{1}{3}}\left(u_{a_{s}}^{R} d_{b_{j}}^{R} u_{c_{k}}^{L}+u_{a_{s}}^{R} d_{b_{j}}^{L} u_{c_{k}}^{R}+u_{a_{s}}^{L} d_{b_{j}}^{R} u_{c_{k}}^{R}\right) \epsilon_{a b c} f(s, j, k), \\
B_{4}(3 / 2)=u_{a_{s}}^{R} d_{b_{j}}^{R} u_{c_{k}}^{R} \epsilon_{a b c} f(s, j, k),
\end{array}\right.
\end{gathered}
$$

where $\epsilon_{a b c}$ is the Levi-Civita tensor in the color indices. $f(s, j, k)$ is a function of the spin indices; but they are connected with the total angular momentum of the nucleon and at this step we do not perform any summation over $s, j$, and $k$. We have denoted with $B_{r}\left(\chi_{z}\right)$ the baryon field in the $S U(2)_{C S}$ irreducible representation of $\operatorname{dim} r=2 \chi+1$ with the chiral spin $\chi$ and its projection $\chi_{z}$.

Now we apply Eqs. (26)-(28) to nucleon interpolators with isospin $1 / 2$, the total angular momentum $J=1 / 2$ with spin zero diquark. The structure of these nucleon interpolators is [14]

$$
N_{ \pm}^{(i)}=\epsilon_{a b c} \mathcal{P}_{ \pm} \Gamma_{1}^{(i)} u_{a}\left(u_{b}^{T} \Gamma_{2}^{(i)} d_{c}-d_{b}^{T} \Gamma_{2}^{(i)} u_{c}\right),
$$

where $\mathcal{P}_{ \pm}=\frac{1}{2}\left(\mathbb{1} \pm \gamma_{4}\right)$. The Dirac structures of $\Gamma_{1}^{(i)}$ and $\Gamma_{2}^{(i)}$ are reported in Table I.

Now if we want to write the $N_{ \pm}^{(i)} \mathrm{s}$ as linear combination of the $B_{r}\left(\chi_{z}\right)$ we need to find the proper expression for $f(s, j, k)$ in Eqs. (26)-(28). At first we note that the diquark in Eq. (29) has spin zero and it does not carry any spin index. Conversely, since $N_{ \pm}^{(i)}$ has spin 1/2, then it carries a spin index given by the quark $u_{a}$. This means that in Eqs. (26)-(28) we need to sum over $j$ and $k$, but not over $s$. $C=i \gamma_{2} \gamma_{4}=\sigma_{2} \otimes \sigma_{3}$, where the Pauli matrix $\sigma_{2}$ acts in the quark spin space. Therefore, we can replace $f(s, j, k) \rightarrow$ $\left(\sigma_{2}\right)_{j k}$ in Eqs. (26)-(28) and sum over $j$ and $k$ but not over $s$.

TABLE I. List of Dirac structures for the $N$ baryon fields with scalar or pseudoscalar diquarks, where $I$ is the isospin and $J^{P}$ indicates spin and parity.

\begin{tabular}{lccc}
\hline \hline$I, J^{P}$ & $\Gamma_{1}^{(i)}$ & $\Gamma_{2}^{(i)}$ & $i$ \\
\hline & $\mathbb{1}$ & $C \gamma_{5}$ & 1 \\
$N^{(i)}\left(\frac{1}{2}^{2} \frac{1}{2}^{ \pm}\right)$ & $\gamma_{5}$ & $C$ & 2 \\
& $i \mathbb{1}$ & $C \gamma_{5} \gamma_{4}$ & 3 \\
& $i \gamma_{5}$ & $C \gamma_{4}$ & 4 \\
\hline \hline
\end{tabular}

In this case $B_{r}\left(\chi_{z}\right)$ will represent baryon interpolators with spin $1 / 2$, in which the diquark has spin zero.

Finally, we can rewrite the nucleon fields in Eq. (29) for each $i$ as

$$
\begin{aligned}
& N_{ \pm}^{(1)}=-\mathcal{P}_{ \pm}\left[\left(B_{4}(-3 / 2) \pm B_{4}(3 / 2)\right)\right. \\
& +\sqrt{\frac{1}{6}}\left(B_{2_{2}}(1 / 2) \mp B_{2_{2}}(-1 / 2)\right) \\
& +\sqrt{\frac{1}{2}}\left(B_{2_{1}}(1 / 2) \mp B_{2_{1}}(-1 / 2)\right) \\
& \left.+\sqrt{\frac{1}{3}}\left(B_{4}(1 / 2) \pm B_{4}(-1 / 2)\right)\right], \\
& N_{ \pm}^{(2)}=\mathcal{P}_{ \pm}\left[-\left(B_{4}(-3 / 2) \pm B_{4}(3 / 2)\right)\right. \\
& +\sqrt{\frac{1}{6}}\left(B_{2_{2}}(1 / 2) \mp B_{2_{2}}(-1 / 2)\right) \\
& +\sqrt{\frac{1}{3}}\left(B_{4}(1 / 2) \pm B_{4}(-1 / 2)\right) \\
& \left.+\sqrt{\frac{1}{2}}\left(B_{2_{1}}(1 / 2) \mp B_{2_{1}}(-1 / 2)\right)\right], \\
& N_{ \pm}^{(3)}=-i \mathcal{P}_{ \pm}\left[\sqrt{\frac{1}{6}}\left(B_{2_{2}}(-1 / 2) \mp B_{2_{2}}(1 / 2)\right)\right. \\
& +\frac{2}{\sqrt{3}}\left(B_{4}(-1 / 2) \pm B_{4}(1 / 2)\right) \\
& \left.+\sqrt{\frac{1}{2}}\left(B_{2_{1}}(-1 / 2) \mp B_{2_{1}}(1 / 2)\right)\right], \\
& N_{ \pm}^{(4)}=i \mathcal{P}_{ \pm}\left[-\sqrt{\frac{3}{2}}\left(B_{2_{2}}(-1 / 2) \mp B_{2_{2}}(1 / 2)\right)\right. \\
& \left.+\sqrt{\frac{1}{2}}\left(B_{2_{1}}(-1 / 2) \mp B_{2_{1}}(1 / 2)\right)\right],
\end{aligned}
$$

where $B_{r}\left(\chi_{z}\right)$ are 


$$
\begin{aligned}
& B_{2_{1}}(-1 / 2)=\epsilon_{a b c} \frac{1}{\sqrt{2}}\left(\frac{\mathbb{1}-\gamma_{5}}{2}\right)\left[\gamma_{4} u_{a}\left\{d_{b}^{T} C\left(\frac{\mathbb{1}-\gamma_{5}}{2}\right) u_{c}\right\}\right. \\
& \left.+u_{a}\left\{d_{b}^{T} C \gamma_{4}\left(\frac{\mathbb{1}-\gamma_{5}}{2}\right) u_{c}\right\}\right] \text {, } \\
& B_{2_{1}}(1 / 2)=\epsilon_{a b c} \frac{1}{\sqrt{2}}\left(\frac{\mathbb{1}-\gamma_{5}}{2}\right)\left[u_{a}\left\{d_{b}^{T} C\left(\frac{\mathbb{1}+\gamma_{5}}{2}\right) u_{c}\right\}\right. \\
& \left.+\gamma_{4} u_{a}\left\{d_{b}^{T} C \gamma_{4}\left(\frac{\mathbb{1}+\gamma_{5}}{2}\right) u_{c}\right\}\right] \text {, } \\
& B_{2_{2}}(-1 / 2)=\epsilon_{a b c}\left(\frac{\mathbb{1}-\gamma_{5}}{2}\right)\left[-\sqrt{\frac{2}{3}} u_{a}\left\{d_{b}^{T} C \gamma_{4}\left(\frac{\mathbb{1}+\gamma_{5}}{2}\right) u_{c}\right\}\right. \\
& -\sqrt{\frac{1}{6}} u_{a}\left\{d_{b}^{T} C \gamma_{4}\left(\frac{\mathbb{1}-\gamma_{5}}{2}\right) u_{c}\right\} \\
& \left.+\sqrt{\frac{1}{6}} \gamma_{4} u_{a}\left\{d_{b}^{T} C\left(\frac{\mathbb{1}-\gamma_{5}}{2}\right) u_{c}\right\}\right], \\
& B_{2_{2}}(1 / 2)=\epsilon_{a b c}\left(\frac{\mathbb{1}-\gamma_{5}}{2}\right)\left[\sqrt{\frac{1}{6}} u_{a}\left\{d_{b}^{T} C\left(\frac{\mathbb{1}+\gamma_{5}}{2}\right) u_{c}\right\}\right. \\
& -\sqrt{\frac{1}{6}} \gamma_{4} u_{a}\left\{d_{b}^{T} C \gamma_{4}\left(\frac{1+\gamma_{5}}{2}\right) u_{c}\right\} \\
& \left.-\sqrt{\frac{2}{3}} \gamma_{4} u_{a}\left\{d_{b}^{T} C \gamma_{4}\left(\frac{\mathbb{1}-\gamma_{5}}{2}\right) u_{c}\right\}\right] \text {, } \\
& B_{4}(-3 / 2)=-\epsilon_{a b c}\left(\frac{\mathbb{1}-\gamma_{5}}{2}\right) u_{a}\left\{d_{b}^{T} C\left(\frac{\mathbb{1}-\gamma_{5}}{2}\right) u_{c}\right\} \text {, } \\
& B_{4}(-1 / 2)=\sqrt{\frac{1}{3}} \epsilon_{a b c}\left(\frac{\mathbb{1}-\gamma_{5}}{2}\right)\left[-u_{a}\left\{d_{b}^{T} C \gamma_{4}\left(\frac{\mathbb{1}+\gamma_{5}}{2}\right) u_{c}\right\}\right. \\
& +u_{a}\left\{d_{b}^{T} C \gamma_{4}\left(\frac{\mathbb{1}-\gamma_{5}}{2}\right) u_{c}\right\} \\
& \left.-\gamma_{4} u_{a}\left\{d_{b}^{T} C\left(\frac{\mathbb{1}-\gamma_{5}}{2}\right) u_{c}\right\}\right] \text {, } \\
& B_{4}(1 / 2)=\sqrt{\frac{1}{3}} \epsilon_{a b c}\left(\frac{\mathbb{1}-\gamma_{5}}{2}\right)\left[\gamma_{4} u_{a}\left\{d_{b}^{T} C \gamma_{4}\left(\frac{\mathbb{1}-\gamma_{5}}{2}\right) u_{c}\right\}\right. \\
& -\gamma_{4} u_{a}\left\{d_{b}^{T} C \gamma_{4}\left(\frac{1+\gamma_{5}}{2}\right) u_{c}\right\} \\
& \left.+u_{a}\left\{d_{b}^{T} C\left(\frac{\mathbb{1}+\gamma_{5}}{2}\right) u_{c}\right\}\right] \\
& B_{4}(3 / 2)=\epsilon_{a b c}\left(\frac{\mathbb{1}-\gamma_{5}}{2}\right) \gamma_{4} u_{a}\left\{d_{b}^{T} C\left(\frac{\mathbb{1}+\gamma_{5}}{2}\right) u_{c}\right\} \text {, }
\end{aligned}
$$

and the brace brackets mean antisymmetrization in the flavor space, e.g., $\left\{d_{b}^{T} C u_{c}\right\}=d_{b}^{T} C u_{c}-u_{b}^{T} C d_{c}$. The parity projector $\mathcal{P}_{ \pm}$in Eq. (30) can be replaced with $\mathcal{P}_{ \pm} \rightarrow \mathbb{1} / \sqrt{2}$, since in the computation of the correlators the presence of $\mathcal{P}_{ \pm}$is irrelevant.

Hence, we have rewritten the nucleon interpolators $N_{ \pm}^{(i)}$ in terms of interpolators that transform according to the irreducible representations of $S U(2)_{C S}$.
The cross-correlation matrix for interpolators $B_{r}\left(\chi_{z}\right)$ and $B_{r \prime}\left(\chi^{\prime} z_{z}\right)$ is

$$
C(t)_{\chi, \chi^{\prime}, \chi_{z}, \chi^{\prime} z}=\left\langle 0\left|B_{r}\left(\chi_{z} ; t\right) B_{r \prime}\left(\chi^{\prime} z_{z} ; 0\right)^{\dagger}\right| 0\right\rangle .
$$

The $S U(2)_{C S}$ restoration requires that the cross-correlators of operators from different representations of $S U(2)_{C S}$ vanish and the cross-correlators of operators within a given representation of $S U(2)_{C S}$ that are diagonal in indices $\chi_{z}$ and $\chi^{\prime}{ }_{z}$ coincide while the off-diagonal vanish. In this case, the diagonal correlators $C(t)_{N_{ \pm}^{(i)}}$ of nucleon interpolators $N_{ \pm}^{(i)}$ from Table I are given as

$$
\begin{aligned}
C(t)_{N_{ \pm}^{(i)}}= & \frac{2}{3} C(t)_{3 / 2}+\frac{1}{12} C(t)_{1 / 2_{2}}+\frac{1}{4} C(t)_{1 / 2_{1}} \\
& \text { for } i=1,2,3 \\
C(t)_{N_{ \pm}^{(4)}}= & \frac{1}{4} C(t)_{1 / 2_{2}}+\frac{3}{4} C(t)_{1 / 2_{1}} .
\end{aligned}
$$

Here $C(t)_{3 / 2}$ is a correlator $C(t)_{\chi, \chi^{\prime}, \chi_{z}, \chi_{z}^{\prime}}$ with $\chi=\chi^{\prime}=3 / 2$ and with any $\chi_{z}=\chi^{\prime}{ }_{z}$, and similar for $C(t)_{1 / 2_{1}}$ and $C(t)_{1 / 2_{2}}$. Hence, in the $S U(2)_{C S}$-symmetric regime all correlators $C(t)_{N_{ \pm}^{(i)}}$ with $i=1,2,3$ should be degenerate. Such a degeneracy was indeed observed at zero temperature upon truncation of the near-zero modes of the Dirac operator in Ref. [14].

\section{CONCLUSIONS}

In this paper we have demonstrated that a chirally symmetric parity-doublet Lagrangian is not only chirally symmetric, but in addition is $S U(2)_{C S}$ and $S U\left(2 N_{F}\right)$ invariant. Then we have constructed nucleon three-quark interpolators that transform under irreducible representations of $S U(2)_{C S}$. Such interpolators are required for lattice studies to establish the chiral spin symmetry.

\section{ACKNOWLEDGMENTS}

This work is supported by the Austrian Science Fund FWF through Grants No. DK W1203-N16 and No. P26627-N27. The authors thank Christian Rohrhofer for cross-checking of all equations of Sec. IV.

\section{APPENDIX CHIRAL TRANSFORMATION OF THE PARITY-DOUBLET LAGRANGIAN}

We begin with the Lagrangian (2.36) of [3] with two Dirac fermions

$\mathcal{L}=i \bar{\psi}_{1} \gamma_{\mu} \partial^{\mu} \psi_{1}+i \bar{\psi}_{2} \gamma_{\mu} \partial^{\mu} \psi_{2}-m\left(\bar{\psi}_{1} \psi_{2}+\bar{\psi}_{2} \psi_{1}\right)$,

which is invariant under chiral transformation with the "mirror assignment": 


$$
\psi_{1} \rightarrow \exp \left(i \alpha \gamma_{5}\right) \psi_{1}, \quad \psi_{2} \rightarrow \exp \left(-i \alpha \gamma_{5}\right) \psi_{2} .
$$

From this we can obtain the chiral transformation of the doublet (4)

$$
\Psi=\left(\begin{array}{l}
\Psi_{+} \\
\Psi_{-}
\end{array}\right)
$$

where

$$
\begin{aligned}
& \Psi_{+}=\frac{1}{\sqrt{2}}\left(\psi_{1}+\psi_{2}\right), \\
& \Psi_{-}=\frac{1}{\sqrt{2}}\left(\gamma_{5} \psi_{1}-\gamma_{5} \psi_{2}\right) .
\end{aligned}
$$

Then upon the "mirror" transformation (A2) the parity doublet (A3) transforms as

$$
\left(\begin{array}{l}
\Psi_{+} \\
\Psi_{-}
\end{array}\right) \rightarrow \exp \left(i \alpha \sigma^{1}\right)\left(\begin{array}{l}
\Psi_{+} \\
\Psi_{-}
\end{array}\right)
$$

Extending the $U(1)_{A}$ mirror transformation (A2) to the axial part of the mirror $S U(2)_{L} \times S U(2)_{R}$ transformation

$$
\begin{aligned}
& \psi_{1} \rightarrow \exp \left(i \frac{\alpha^{a} \tau^{a}}{2} \gamma^{5}\right) \psi_{1}, \\
& \psi_{2} \rightarrow \exp \left(-i \frac{\alpha^{a} \tau^{a}}{2} \gamma^{5}\right) \psi_{2},
\end{aligned}
$$

we obtain the axial part of the $S U(2)_{L} \times S U(2)_{R}$ transformation of the parity doublet (6):

$$
\left(\begin{array}{c}
\Psi_{+} \\
\Psi_{-}
\end{array}\right) \rightarrow \exp \left(i \frac{\alpha^{a} \tau^{a}}{2} \otimes \sigma^{1}\right)\left(\begin{array}{l}
\Psi_{+} \\
\Psi_{-}
\end{array}\right)
$$

[1] B. W. Lee, Chiral Dynamics (Gordon and Breach, Philadelphia, 1972).

[2] C. E. Detar and T. Kunihiro, Linear $\sigma$ model with parity doubling, Phys. Rev. D 39, 2805 (1989).

[3] D. Jido, M. Oka, and A. Hosaka, Chiral symmetry of baryons, Prog. Theor. Phys. 106, 873 (2001).

[4] L. Y. Glozman, Restoration of chiral and U(1)A symmetries in excited hadrons, Phys. Rep. 444, 1 (2007).

[5] L. Olbrich, M. Ztnyi, F. Giacosa, and D. H. Rischke, Threeflavor chiral effective model with four baryonic multiplets within the mirror assignment, Phys. Rev. D 93, 034021 (2016); S. Gallas, F. Giacosa, and D. H. Rischke, Vacuum phenomenology of the chiral partner of the nucleon in a linear sigma model with vector mesons, Phys. Rev. D 82, 014004 (2010).

[6] C. Sasaki, Parity doubling of baryons in a chiral approach with three flavors, Nucl. Phys. A970, 388 (2018).

[7] C. Rohrhofer, Y. Aoki, G. Cossu, H. Fukaya, L. Y. Glozman, S. Hashimoto, C. B. Lang, and S. Prelovsek, Approximate degeneracy of $J=1$ spatial correlators in high temperature QCD, Phys. Rev. D 96, 094501 (2017).
[8] L. Y. Glozman, SU(4) symmetry of the dynamical QCD string and genesis of hadron spectra, Eur. Phys. J. A 51, 27 (2015).

[9] L. Y. Glozman and M. Pak, Exploring a new SU(4) symmetry of meson interpolators, Phys. Rev. D 92, 016001 (2015).

[10] L. Y. Glozman, Chiralspin symmetry and the phase diagram of QCD, arXiv:1712.05168.

[11] M. Denissenya, L. Y. Glozman, and C. B. Lang, Symmetries of mesons after unbreaking of chiral symmetry and their string interpretation, Phys. Rev. D 89, 077502 (2014).

[12] M. Denissenya, L. Y. Glozman, and C. B. Lang, Isoscalar mesons upon unbreaking of chiral symmetry, Phys. Rev. D 91, 034505 (2015).

[13] M. Denissenya, L. Y. Glozman, and M. Pak, Evidence for a new $S U(4)$ symmetry with $J=2$ mesons, Phys. Rev. D 91, 114512 (2015).

[14] M. Denissenya, L. Y. Glozman, and M. Pak, Emergence of a new $S U(4)$ symmetry in the baryon spectrum, Phys. Rev. D 92, 074508 (2015); Erratum 92, 099902 (2015).

[15] C. B. Lang, Low lying eigenmodes and meson propagator symmetries, Phys. Rev. D 97, 114510 (2018). 